\title{
Spokes in high power impulse magnetron sputtering plasmas
}

\author{
Ante Hecimovic $\Varangle \S$ and Achim von Keudell \\ Institute for Experimental Physics II, Ruhr-University Bochum, Germany
}

\begin{abstract}
High power impulse magnetron sputtering (HiPIMS) is a deposition technique where a metal magnetron target is sputtered in a high density plasma to synthesize thin layers with superior properties on a substrate material. These plasmas are characterised by short pulses in the range of $50 \mu \mathrm{s}$ to $200 \mu \mathrm{s}$ and very high peak powers in the range of several $\mathrm{kW} / \mathrm{cm}^{2}$ per target area. The understanding of these dynamic plasmas is of upmost interest for the further development of this coating technique. Fast camera measurements revealed the formation of localised ionisation zones in these plasmas that propagate with a velocity of the order $\mathrm{km} / \mathrm{s}$. In the case of a circular magnetron, the movement of these ionisation zones appears like the movement of a set of spokes, which led to this common expression spoke to illustrate the pattern formation in these high density plasmas. The analysis and understanding of the spoke phenomenon is still a matter of an open debate, because the analysis and the theoretical description is hampered by the inherent complexity of these plasmas. In this paper, we review the experimental observations of the spoke phenomenon and highlight several approaches for their theoretical explanation.
\end{abstract}

$\ddagger$ present address: Max-Planck Institute for Plasma Physics, Garching, Germany

$\S$ Email corresponding author: ante.hecimovic@ipp.mpg.de 


\section{Introduction}

High power impulse magnetron sputtering (HiPIMS) plasmas belong to the class of magnetron sputtering discharges that have been originally developed in the 1970s as a deposition tool. Since the 1990s different variations of the power management of these magnetron sputtering have been explored, such as bi-polar and unipolar pulsed dc (pulsed dcMS), dual cathode mid-frequency pulsing and more recently high power impulse magnetron sputtering (HiPIMS). The latter technique provides high plasma power-densities at the target, in the range of $\mathrm{kW} / \mathrm{cm}^{2}$, through the application of low duty cycle voltage pulses of typical length of $50 \mu$ s to $200 \mu$ s while maintaining a low average power to avoid any overheating of the target. As a result, much higher ionisation degrees up to $100 \%$ were achievable for plasmas operated at a duty cycle of $1 \%$ or less. The coatings deposited by these techniques have shown excellent properties, which generated an increased interest in these deposition methods and initiated fundamental research on the nature of HiPIMS plasmas.

In magnetrons, the magnetic field is created by permanent magnets placed behind a cathode (commonly referred to as target) generating a closed magnetic field arch above the target. The strength of the magnetic fields of a magnetron is typically up to several $100 \mathrm{mT}$, which is sufficient to magnetically confine the light electrons, whereas the heavy ions are not affected by the magnetic field. The confined electrons will experience several drifts, such as an $\mathbf{E} \times \mathbf{B}$ drift, a gradient magnetic field drift $(\nabla \mathbf{B})$, a pressure (density) gradient (diamagnetic drift), and a curvature magnetic field drift (curvature drift). A typical magnetron consists of a circular target, which then also defines a cylindrical coordinate system with an azimuthal coordinate $\phi$ along the perimeter of the target, a radial coordinate $r$ from the centre of the target to the perimeter of the magnetron, and an axial coordinate $z$ describing the distance normal to the target surface. Due to this confinement, a typical magnetron plasma consist of a torus above a circular magnetron target. The intense sputtering causes local etching generating a so called racetrack as the footprint of the magnetron plasma on the target surface. A typical configuration of planar circular magnetron is shown in figure 1.

The high peak power during the pulse implies very bright plasmas that can be recorded by ICCD cameras using acquisition time of tens of nanoseconds only. Three groups have simultaneously and independently reported plasma inhomogeneities above the cathode in HiPIMS plasmas $[2,3,4]$ that rotate in the $\mathbf{E} \times \mathbf{B}$ direction with velocities in the range of $\mathrm{km} / \mathrm{s}$. These patterns were similar to the so called rotating spokes observed in Hall thrusters [5]. Based on this similarity, the emission patterns are now also coined spokes in the HiPIMS community.

In general, $\mathrm{dc}$ magnetron plasmas fall into the category of low $\beta$ plasmas $(\beta ; 1 \%)$, where $\beta$ is the ratio of kinetic $\left(\mathrm{p}_{k}=\mathrm{n}^{*} \mathrm{k}_{B}{ }^{*} \mathrm{~T}\right)$ to magnetic pressure $\left(\mathrm{p}_{m}=\mathrm{B}^{2} / 2 \mu_{0}\right)$. Low $\beta$ indicates that the plasma density and temperature are low enough that the electrons are still considered magnetically confined. Also in HiPIMS, the $\beta$ value for the plasma at locations directly in the target vicinity is of the order of $1 \%$, but can reach values close 


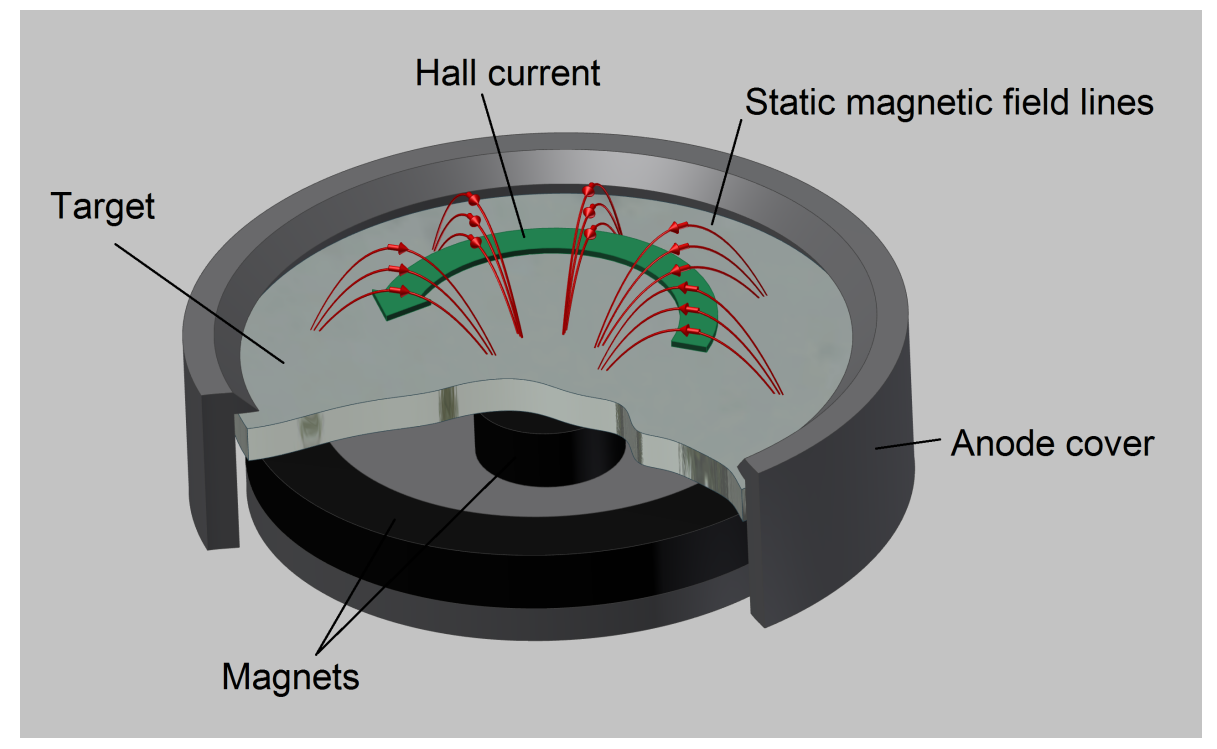

Figure 1. Cross section of planar circular magnetron with indicated static magnetic field lines and electron Hall current, adopted from [1].

to $100 \%$ in the region of the expanding plasma towards the substrate, where the plasma density may still be high, but the magnetic field weaker. This automatically leads to unstable modes triggered by electrostatic instabilities. Self-organisation patterns or nonuniformities are commonly observed in those plasma discharges. Such self-organisation patterns in the form of striations of the positive column have been observed already in the early investigations of a simple dc discharge [6], and since then have been observed in many different plasma configurations [7]. The development of non-uniformities is typically associated with a specific instability mechanism. In a plasma, a single particle might oscillate under the influence of external forces leading to the propagation of waves with an amplitude that may rise indefinitely - an instability occurs. All instabilities are characterised by their dependence on plasma quantities, such as density, electromagnetic fields, or pressure.

The spoke phenomenon belongs to the class of $\mathbf{E} \times \mathbf{B}$ instabilities propagating in the $\mathbf{E} \times \mathbf{B}$ direction in the presence of a density and/or a magnetic field gradient. The spoke phenomenon is a large scale low frequency instability that may be triggered by the Simon-Hoh mechanism [8, 9]. The Simon-Hoh type instability results from the relative motion of electrons and ions in a crossed electric and magnetic field and causes a phase shift in the response of ions and electrons to the perturbations of the electric field in azimuthal direction $E_{\phi}$. These instabilities may also lead a cross-B field electron transport that has been found to be a factor of 5 larger than classical Bohm diffusion $[10]$.

Despite this general connection of the spoke phenomenon to the large class of known plasma instabilities, the analysis and understanding of the spoke phenomenon is still an open debate due to the complexity of the system. The spoke phenomenon may also be influenced by the neutral gas dynamic and the gas rarefaction caused by the strong 
sputter wind, it may also be influenced by secondary electron emission, and it may also be influenced by the strong Hall currents, which modify the static magnetic field dynamically during a HiPIMS pulse, and it may also be influenced by any local target poisoning in a reactive HiPIMS system. Since all these effects may contribute to the spoke phenomenon at the same time their understanding by experiments and theory is very challenging. For example, the spoke phenomenon is a true $3 \mathrm{D}$ plasma equilibrium, which is in most cases beyond the capabilities of current computer codes. Any well known plasma instability from classical textbook plasma physics such as the Simon Hoh instability might not be applicable at the end, because either the perturbation is much stronger or boundary effects from the target surface could be much more important than gradient driven instabilities. A great help in resolving this puzzle, would be the availability of experimental data on detailed plasma parameters inside these spokes. However, due to the high power load, all diagnostics have to be non-invasive which induces ambiguities in the interpretation. For example, there are still no robust values for the plasma density inside a spoke available.

In this review, we discuss the current understanding of the spoke phenomenon from an experimental point overview and describe several theoretical explanations put forward in the community. 


\section{Appearance of spokes}

\subsection{Plasma oscillations in DC magnetron sputtering plasma}

The spoke phenomenon is triggered by an instability that occurs in a high density plasma exhibiting gradients in density and/or magnetic field. Such instabilities are most pronounced in high performance plasmas, but were already observed in traditional low power dc magnetron sputtering plasmas before. A key feature of the magnetron configuration is the presence of a non-uniform magnetic field in front of the cathode. The magnetic field confines the electrons, thus sustaining the plasma operation at low background gas pressures and low voltages. The transport of species in a magnetron discharge is determined by electrons escaping the magnetic trap and crossing the magnetic field lines, the so-called cross-B field diffusion. Semi-empirical estimates of this electron cross-B diffusion by Bohm predicts higher diffusion rates compared to classical diffusion. This led to the expression of anomalous electron transport in magnetron plasmas.

The electron drift in azimuthal direction, perpendicular to the magnetic field, is inherently unstable, as any deviation from the charge neutrality generates azimuthal electric fields $E_{\phi}$ which causes second order $E_{\phi} \times B$ drifts. Such instabilities are known as gradient-drift or neutral-drag instabilities. In 1978, Thornton anticipated the existence of a whole range of instabilities and considered a correlation between these instabilities and the anomalous electron transport. He observed oscillations in a frequency range from 50 to $500 \mathrm{kHz}$ [11]. Later, Sheridan and Goree used Langmuir probes and detected oscillations in DC magnetron plasmas at frequencies between 5 and $200 \mathrm{kHz}$, with density fluctuations up to 5\% [12]. They observed a scaling of the electron confinement time with the mean square electric field fluctuations, however, they concluded that the observed oscillations are probably not responsible for the cross-B transport.

The correlation between the fluctuations and the anomalous transport has been investigated by Martines et al. $[13,14]$ by studying the electrostatic fluctuations in DC magnetron plasmas. The fluctuations have been observed as a set of coherent modes, propagating in the direction of the $\mathbf{E} \times \mathbf{B}$ drift. The power densities were in the range $0.5-6 \mathrm{~W} / \mathrm{cm}^{2}$. They observed a dependence of these fluctuations on discharge power and neutral gas pressure: (i) the fluctuation of the floating potential varies with the power, increasing from $3 \%$ up to $20 \%$, (ii) the ion saturation current fluctuations vary up to

$30 \%$ above the racetrack, rather independently from discharge power; (iii) by increasing the neutral gas pressure to several $\mathrm{Pa}$, a progressive transition towards a turbulent state is observed; (iv) finally, the fluctuations are not simultaneously present at all times. The authors attempted to explain these fluctuations as coupled oscillators, transferring energy between different modes in a stochastic manner. 


\subsection{Spoke in metal HiPIMS plasmas}

The spoke phenomenon is most visible in HiPIMS plasmas. Due to their pulsing nature, a HiPIMS plasma passes through three different stages in each pulse: (1) ignition, (2) development, and (3) quasi steady state, if the pulse is long enough. The HiPIMS plasmas are transient plasmas compared to the steady state plasma during DC magnetron sputtering. Three groups have simultaneously and independently used ICCD camera imaging facing the magnetron surface of HiPIMS plasmas and have reported plasma inhomogeneities above the cathode $[2,3,4]$ that rotate in the $\mathbf{E} \times \mathbf{B}$ direction with velocities in the range of $\mathrm{km} / \mathrm{s}$.
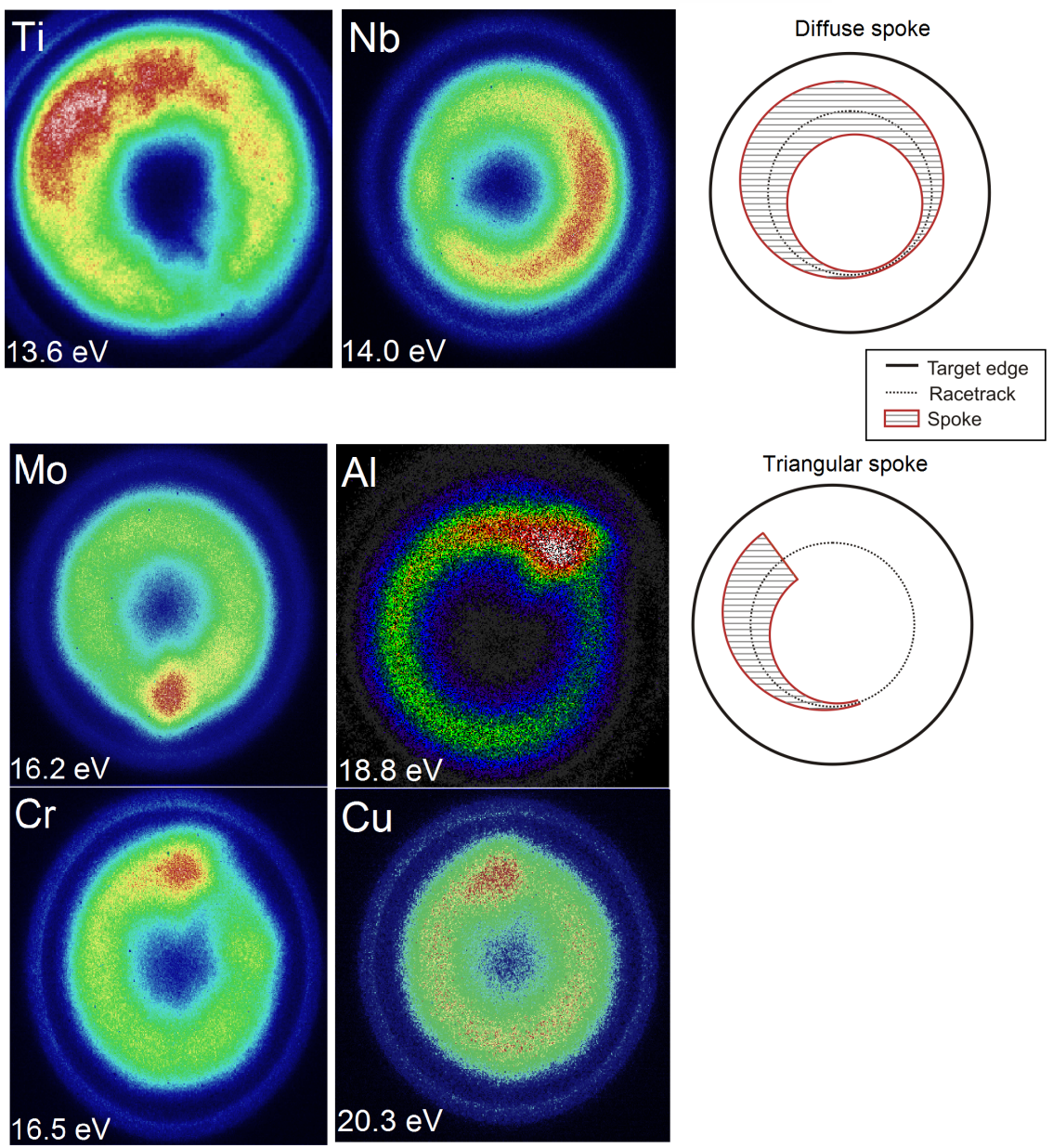

Figure 2. The shape of spokes for different target materials, and a sketch of the diffuse and triangular spoke, adopted from [15]. Magnetron is 2" round planar magnetron

It has been observed that the shape of the spokes [15], i.e. the emission distribution, strongly depends on the target material, as shown in figure 2. Two typical shapes of spokes have been observed: the diffuse shape characterised by a pronounced emission maximum with a diffuse leading and trailing edge, and the triangular shape with a narrow leading edge and a broad and sharp trailing edge. The comparison of the light 
intensity along the racetrack and the ion saturation current measured at the magnetron perimeter reveals that the plasma in such a spoke spreads over the racetrack in the case of a diffuse shape, whereas the plasma is very much localised in the case of a triangular shape. Based on this comparison, one can use the ICCD images as a qualitative measure of the electron density distribution.

The azimuthal distribution of the species emission in the spoke was recorded by mounting bandpass interference filters in front of the camera lens [16, 17]. In the following, the observation for an $\mathrm{Al}$ target are presented. For triangular spokes above an $\mathrm{Al}$ target, the brightest region of the spoke is dominated by emission from $\mathrm{Ar}$ and from target ions $\left(\mathrm{Al}^{+}\right)$, whereas the emission of target neutrals (Al) is small. Furthermore, the emission due to de-excitation of higher energy levels, especially for ion lines, was also localised in the brightest region of the spokes. Outside of the spoke region, the emission of target neutrals ( $\mathrm{Al}$ ) is dominant, while Ar ion and target ion emission is very low. Based on the brightness of the spokes, one may conclude that the spoke region is also a dominant ionisation zone consistent with observation of strong ion lines inside the spokes. The emission from target atoms and ions originates mainly from a region within less than $3 \mathrm{~mm}$ from the target surface, whereas emission from Ar atoms and ions originates from a region further apart from the target surface.

The azimuthal distribution of sputtered neutrals $(\mathrm{Ti})$ and ions $\left(\mathrm{Ti}^{+}\right)$in the ground state has been determined using laser induced fluorescence (LIF) for the case of a $\mathrm{Ti}$ target [18]. In addition, the densities of Ar metastable are recorded. It was observed that ground states of Ti neutrals as well as Ar metastables are significantly depleted along the racetrack and no correlation between this depletion and the spoke patterns could be established. However, the ground state of $\mathrm{Ti}^{+}$ions was depleted inside a spoke at the expense of higher excited states and multiple charged ions. This indicates the occurrence of a very strong ionisation inside a spoke due to the presence of hot electrons.

Ni et al. [19] mounted a streak camera along the axial direction and observed the expansion of the HiPIMS revealing complex structures of the spoke: the $y$ axis of the streak camera image refers to the azimuthal position of the plasma emission recorded through a slit, while the $x$ axis corresponds to the time. These streak camera images revealed that spokes are moving along the targets racetrack in $\mathbf{E} \times \mathbf{B}$ direction but also eject plasma flares in azimuthal direction. The flare velocity in axial direction was determined to be about $20 \mathrm{~km} / \mathrm{s}$. The flare velocity was lower at the beginning of the pulse when the discharge current was low and the plasma was less dense, compared to later phases within the pulse. Such flares may indicate electrons leaving the closed drift region.

The diffuse spoke shape is observed for target elements exhibiting low second ionisation potentials, whereas triangular spokes are observed for target elements with high second ionisation potential [15]. This distinction is surprising, but can be explained by the nature of secondary electron (SE) generation at the target that sustains the plasma: in order to generate a secondary electron upon impact of an ion from the plasma, the ionisation potential of this ion has to be at least two times higher than the 
work function of the target material. This condition is not fulfilled for singly charged, but for doubly charged metal ions (and $\mathrm{Ar}^{+}$ions). At very high plasma powers, the plasma gas Ar is significantly rarefied by the sputter wind so that secondary electron generation can only be sustained by doubly charged metal ions. The leading edge of the spoke is a region of stronger ambipolar diffusion of electrons and ions away from the target that, as confirmed by probe data. The nature of the trailing edge of a spoke, however, is governed by the balance between loss of plasma species and generation of plasma species by secondary electrons: (i) target elements exhibiting low second ionisation potentials (implying a low efficiency for SE generation) are characterised by sharp ends of the spokes, being parallel to the magnetic field lines. Apparently the plasma reaches open magnetic field lines and may travel towards the substrate - flares are observed. This loss of plasma cannot be be compensated by plasma generation due to the inefficient secondary electron generation at the target surface; (ii) target elements exhibiting high second ionisation potential (implying a high efficiency for SE generation) are characterised by diffuse ends of the spokes where the plasma may still reach open magnetic field lines causing transport towards the target. But this loss at the trailing end of the spoke is compensated by the efficient and continuous generation of plasma by secondary electrons.

\subsection{Spokes in reactive HiPIMS plasmas}

As described in previous section, the spokes shape is closely correlated to the properties of the species in the target vicinity, and their ability to generate secondary electrons at the target. Addition of a reactive gas $\left(\mathrm{N}_{2}\right.$ or $\left.\mathrm{O}_{2}\right)$ changes both the target surface composition and the gas composition in the target vicinity, as described in great detail elsewhere [20]. At very low concentrations of reactive gas, the discharge is considered to be in a metallic mode (M). Increasing the reactive gas concentration, the target starts to become coated by a compound layer due to the reaction of reactive gas species with the target surface. At moderate reactive gas concentrations, the plasma is considered to be in the transition mode $(\mathrm{T})$, but enters in the so-called poisoned mode $(\mathrm{P})$ if the complete target is covered by the compound. This transition can be followed by optical diagnostics as a transition from metal to reactive gas dominated emission [21].

It is interesting to note that a diffuse spoke is observed in the poisoned mode irrespective of the chosen combination of target and reactive gas admixture $\left(\mathrm{Al} / \mathrm{N}_{2}\right.$, $\mathrm{Al} / \mathrm{O}_{2}, \mathrm{Cr} / \mathrm{N}_{2}, \mathrm{Cr} / \mathrm{O}_{2}, \mathrm{Ti} / \mathrm{N}_{2}, \mathrm{Ti} / \mathrm{O}_{2}$ ), with two examples shown in figure 3 [21]. This

finding is consistent with the secondary electron production at a poisoned target surface in general that is much higher $[22,23]$ compared to a metal target. Thereby, the plasma loss at the trailing edge of a spoke can be compensated and a diffuse shape emerges. The transition to a poisoned mode also leads to a decrease of the discharge voltage required to sustain the plasma at fixed current. 

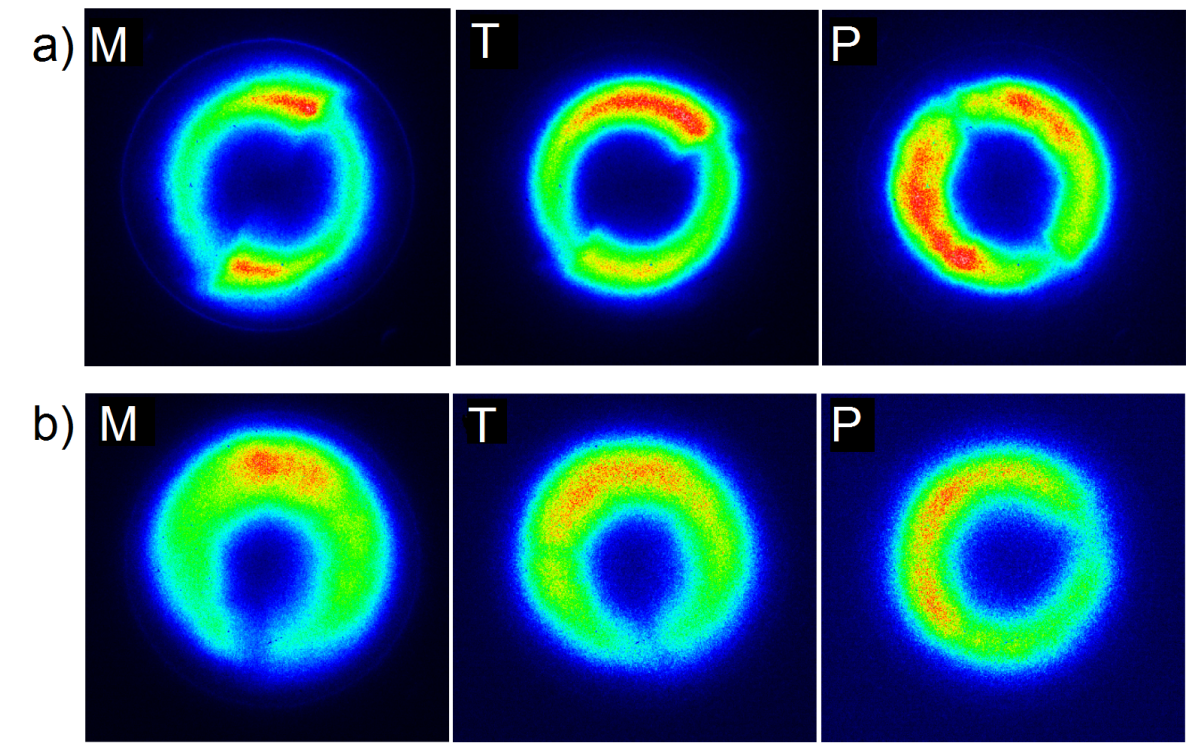

Figure 3. Shape of the spoke in metallic mode $\mathbf{M}$, transition mode $\mathbf{T}$, and poisoned mode $\mathbf{P}$. a) $\mathrm{Al}$ target in $\mathrm{Ar} / \mathrm{O}_{2}$ gas mixture. b) Ti target in $\mathrm{Ar} / \mathrm{N}_{2}$ gas mixture, adopted from [21].

\subsection{Spokes in HiPIMS plasmas on rectangular magnetrons}

For industrial applications, rectangular magnetrons are commonly used. The ICCD imaging of the spokes on a rectangular magnetron shows elongated spokes on the straight part of the racetrack, and "bunching" of spokes in the curved parts of the racetrack, as shown in figure 4 . This observation can be correlated to the change in the magnetic field configuration in the corners compared to the straight parts of the rectangular target. Hnilica et al. have shown that increasing the magnetic field strength increases the spoke mode number [24], which is consistent with the observation of the spoke "bunching" in the curved parts of the magnetron. An increase in plasma power may cause the length of the spokes to become shorter in case of chromium or longer in case of titanium as target material. An increase in pressure does not affect the shape of the spokes, but the length of the spokes becomes shorter [25, 26, 27, 28]. 


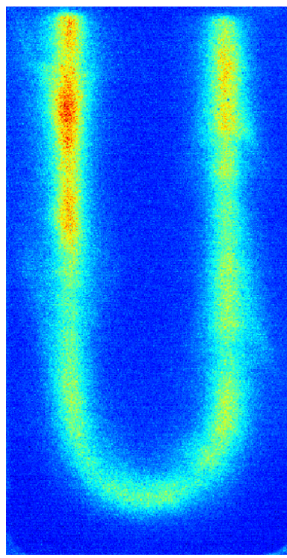

a)

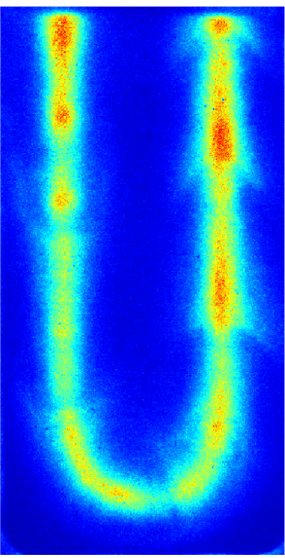

b)

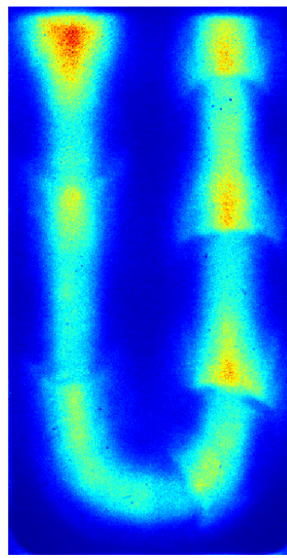

c)

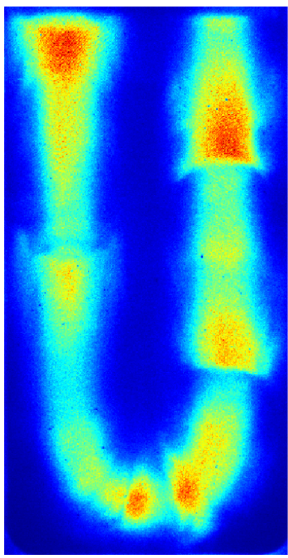

d)

Figure 4. The spokes on rectangular magnetron with $\mathrm{Ti}$ target for different peak current densities: a) $0.2 \mathrm{~A} / \mathrm{cm}^{2}$, b) $0.7 \mathrm{~A} / \mathrm{cm}^{2}$, c) $1.8 \mathrm{~A} / \mathrm{cm}^{2}$, ) $2.9 \mathrm{~A} / \mathrm{cm}^{2}$, adopted from [25]. Rectangular planar magnetron, $90 \mathrm{~mm}$ x $250 \mathrm{~mm}$ 


\section{Properties of spokes}

The spokes are characterised with respect to their rotation direction and velocity, and their mode number (number of spokes along the racetrack).

\subsection{Spoke rotation direction}

The rotation direction exhibits a strong dependence on power density and pressure, as measured by Yang et al. [29], illustrated in figure 5. The exact values of power and pressure where the rotation direction changes depend on the geometry of the magnetron, with the shape and magnitude of the magnetic field having the most significant influence. In the following, the $\mathbf{E} \times \mathbf{B}$ direction is defined by the $E$-field in the pre-sheath pointing towards the target and the $B$-field running in radial direction parallel to the target above the racetrack.

The observed trends regarding the rotation direction can be separated into four phases: Phase I corresponds to the low current regime, where the spokes rotate in the opposite direction to the $\mathbf{E} \times \mathbf{B}$ drift, i.e. retrograde $\mathbf{E} \times \mathbf{B}$ motion; Phase II corresponds to a reversal of the spoke rotation from retrograde $\mathbf{E} \times \mathbf{B}$ motion to $\mathbf{E} \times$ $\mathbf{B}$ motion; Phase III corresponds to an $\mathbf{E} \times \mathbf{B}$ motion with a decrease in the spoke velocity at high discharge currents; Phase $I V$ corresponds to the disappearance of any observable self-organisation patterns. The plasma becomes homogeneous.

At very low power densities below $0.1 \mathrm{~W} / \mathrm{cm}^{2}$ (current of $\sim 10 \mathrm{~mA}$ at 2 " planar target), it has been observed that the spoke rotation direction alternates on time scales of about $1 \mathrm{~ms}$. In the power density range from 0.1 to $1 \mathrm{~W} / \mathrm{cm}^{2}(\sim 10 \mathrm{~mA}-100 \mathrm{~mA}$ at 2" planar target), the spoke rotation direction is opposite to the expected electron $\mathbf{E}$ $\times \mathbf{B}$ drift (retrograde $\mathbf{E} \times \mathbf{B}$ rotation). At power densities between $1 \mathrm{~W} / \mathrm{cm}^{2}$ and 25 $\mathrm{W} / \mathrm{cm}^{2}$ ( $100 \mathrm{~mA}-1 \mathrm{~A}$ at 2" planar target), the plasma starts to oscillate, which has been compared to breathing mode observed in Hall thrusters [29]. Further increasing the power, the breathing plasma behaviour turns into disordered oscillations which are characterised by a stochastic behaviour. At power density of about $250 \mathrm{~W} / \mathrm{cm}^{2}(\sim$ 10 A at 2" planar target) the plasma spokes reappear with a spoke rotation in $\mathbf{E} \times$ $\mathbf{B}$ direction. The threshold of the power density at which the transition takes place depends on target material [17], pressure [29], and magnetic field configuration [30]. At peak power density above $2 \mathrm{~kW} / \mathrm{cm}^{2}$ ( $~ 70 \mathrm{~A}$ at 2 " planar target) and for certain combinations of target/background gas (in Ar: Cr, Au, Cu, Al, Ta, Mo, in Kr: Al, and in gasless environment: $\mathrm{Cu}$ ) the spokes disappear and the plasma becomes completely homogeneous. In the homogeneous mode the system exhibits high impedance, as the plasma is dominated by self-sputtering. In this mode, substantially higher ion fluxes have been measured at the substrate $[31,32]$. In reactive HiPIMS, the transition to a homogeneous plasma has not been observed for any target/gas combination. The transition to a homogeneous plasma at very high powers depends also on the target element/background gas combination, and the pressure. 


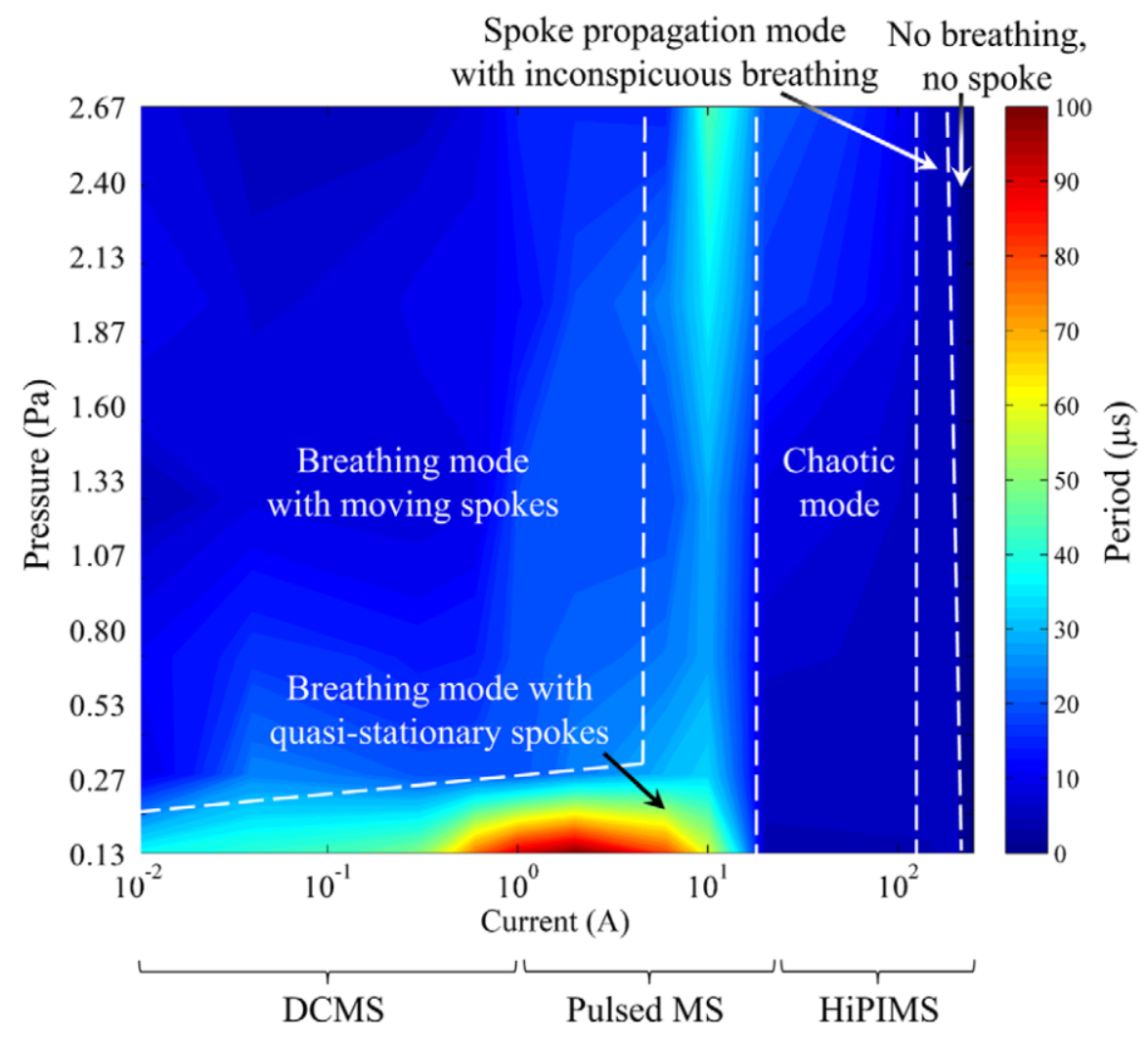

Figure 5. Floating potential characteristic period contour for $\mathrm{Au}$ target, at $\mathrm{Ar}$ pressure from $0.13 \mathrm{~Pa}$ to $2.7 \mathrm{~Pa}$ and current from $10 \mathrm{~mA}$ to $300 \mathrm{~A}$. Modes are indicated as regions separated by white dashed lines, adopted from [29]. Magnetron is 3 " round planar magnetron.

\subsection{Spoke rotation velocity}

The rotation velocity of the spokes has been determined by: (1) comparison of the shift in the signal of two photomultiplier detectors, measuring light emission from two positions along the racetrack [3]; (2) use of streak camera [33], and (3) measurements of the floating potential shift on the azimuthally distributed flat probes [17]. One should keep in mind that the observed spoke motion can be correlated with movement of the ionisation zone (phase velocity), and it does not indicate the movement of the plasma (group velocity).

The rotation velocity of the spokes depends on (1) power density, (2) pressure, and (3) target material. At low powers, below $25 \mathrm{~W} / \mathrm{cm}^{2}(\sim 1$ A at 2 " planar target), the spokes rotate in retrograde $\mathbf{E} \times \mathbf{B}$ direction. In the range $0.1 \mathrm{~W} / \mathrm{cm}^{2}-2 \mathrm{~W} / \mathrm{cm}^{2}$ the velocity increase is proportional to the power density, reaching values up to 2 $\mathrm{km} / \mathrm{s}$, as shown in figure 6 . From $2 \mathrm{~W} / \mathrm{cm}^{2}-250 \mathrm{~W} / \mathrm{cm}^{2}$, the spoke velocity exhibits minor dependence on the power densities and is independent of the target material. Consequently, the dynamic of spokes at low plasma powers is apparently governed by the dynamics of the background gas. This conclusion is consistent with ICCD camera images of a DC plasma for Ar and Ti species that showed in case of dcMS only emission 
from Ar neutrals [17, 34].

a)
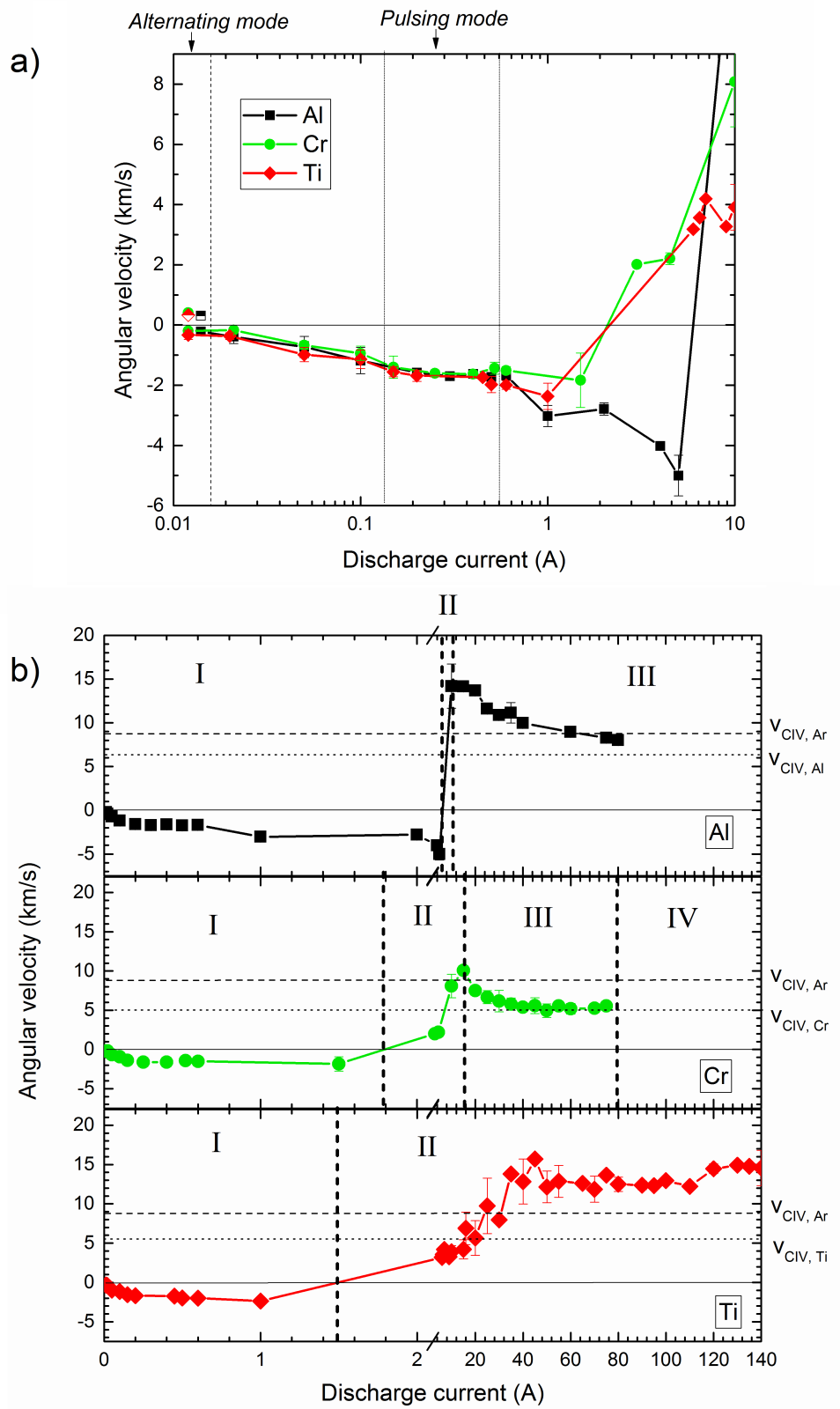

Figure 6. Spoke angular velocity for $\mathrm{Al}, \mathrm{Cr}$ and $\mathrm{Ti}$ targets as a function of discharge current. Magnetron is 2" round planar magnetron. The half-filled symbols stand for $\mathbf{E} \times \mathbf{B}$ rotation velocity for alternating mode at lowest discharge currents. Negative velocities represent retrograde $\mathbf{E} \times \mathbf{B}$ motion. a) Current range $10 \mathrm{~mA}$ to $10 \mathrm{~A}$, all target materials on the same graph. b) Current range 0 to $140 \mathrm{~A}$, The horizontal dashed line denotes the critical ionisation velocity (CIV) for argon $\mathrm{v}_{C I V, A r}$, the horizontal dotted line denotes the CIV for the corresponding metal atoms. Four different phases I-IV denote the discharge current range for retrograde motion (I), direction reversal (II), $\mathbf{E} \times \mathbf{B}$ motion (III), and homogeneous plasma (IV). For details see text. [17]

At peak power density above $25 \mathrm{~W} / \mathrm{cm}^{2}$ ( 1 A at 2 " planar target), the spoke velocity starts exhibiting a strong dependence on the target material. At these powers, 
the density of the sputtered material in the target vicinity becomes comparable to the Ar density, and it is reasonable to conclude that the sputtered material is responsible for the change in the spoke dynamics. This is consistent with a transition from Ar dominated sputtering to self-sputtering, typically observed in HiPIMS plasmas. The key properties of the sputtered atoms influencing the spoke dynamics are ionisation potential, atomic mass, sputter yield, and secondary electron generation. All values are listed in table 1. The influence on the spoke dynamics may occur via the ionisation potential of all elements being lower than the ionisation potential of Ar so that ionisation becomes more efficient due to the high abundance of low energy electrons [35]. In addition, the intense sputter wind causes strong Ar gas rarefaction and secondary electrons enter the plasma at high energies.

\begin{tabular}{l|llllll} 
Element & $\begin{array}{l}\text { First ionisa- } \\
\text { tion potential } \\
(\mathrm{eV})\end{array}$ & $\begin{array}{l}\text { Mass } \\
(\mathrm{amu})\end{array}$ & $\begin{array}{l}\text { Sputter yield } \\
(\text { Ar at } 500 \mathrm{eV}) \\
{[36]}\end{array}$ & $\begin{array}{l}\text { Sputter yield } \\
(\mathrm{SS} \text { at 500 eV) } \\
{[36]}\end{array}$ & $\begin{array}{l}\gamma_{\text {see }} \\
{[37]}\end{array}$ & from \\
\hline $\mathrm{Al}$ & 5.98 & 26.98 & 0.65 & 1.05 & 0.091 \\
\hline $\mathrm{Au}$ & 9.22 & 196.97 & 2.46 & 1.96 & 0.057 \\
\hline $\mathrm{Cr}$ & 6.76 & 51.99 & 1.39 & 1.19 & 0.091 \\
\hline $\mathrm{Cu}$ & 7.72 & 63.55 & 2.46 & 2.10 & 0.082 \\
\hline $\mathrm{Mo}$ & 7.09 & 95.95 & 1.05 & 0.70 & 0.130 \\
\hline $\mathrm{Nb}$ & 6.75 & 92.90 & 0.79 & 0.52 & 0.130 \\
\hline $\mathrm{Ti}$ & 6.83 & 47.86 & 0.63 & 0.52 & 0.114 \\
\hline $\mathrm{Ar}$ & 15.75 & 39.94 & & & \\
\hline
\end{tabular}

Table 1. Characteristic parameter of individual target elements, $\gamma_{s e e}$ is for Ar ion impinging on the target

The spoke velocities are at about $10 \mathrm{~km} / \mathrm{s}$, which is much larger than the spoke velocity in dcMS. The peak velocities reach about $15 \mathrm{~km} / \mathrm{s}$ depending on the target material and background gas pressure. A possible explanation for the spoke velocity is the critical ionisation velocity $(C I V)$ proposed by Brenning et al. [38]: if the kinetic energy of the plasma species moving with respect to the resting neutral gas background is above the ionisation potential of the neutral species, efficient ionisation at the leading edge of a spoke can occur. Based on the simple picture, $v_{C I V}=\sqrt{2 e U / m}$, with $U$ being the ionisation potential of the atom and $m$ the mass of the atom. The predicted $v_{C I V}$ values for different target materials are shown in figure 6 as horizontal dashed lines. One can clearly see that the spoke velocities are in the range of the $v_{C I V}$ values only during HiPIMS operation, but a significant discrepancy between the measured velocities and $v_{C I V}$ remains.

\subsection{Spokes mode number}

At powers below $25 \mathrm{~W} / \mathrm{cm}^{2}$ ( 1 A at 2 " planar target), during dcMS operation, only one spoke has been observed above the racetrack of the 2 " circular target $[34,17]$. On larger 
magnetrons, with $10 \mathrm{~cm}$ diameter, spokes with mode numbers four and five have been observed [39]. At powers around $25 \mathrm{~W} / \mathrm{cm}^{2}$ ( 1 A at 2" planar target), during HiPIMS operation, the plasma behaviour becomes stochastic with the spoke rotation changing from retrograde $\mathbf{E} \times \mathbf{B}$ to $\mathbf{E} \times \mathbf{B}$ rotation. Increasing the power further, the spokes appear again with a spoke mode number of typically four or five. A continuous increase of the current causes the spoke mode number to reduce until one spoke covering the racetrack is established. As mentioned earlier, for some target material/gas combination, the spokes may also disappear completely at very high powers and the plasma becomes homogeneous. We assume that this transition to a homogeneous plasma can occur for all target material/gas combinations at sufficiently high plasma powers. However, this has not been observed, as the maximum power is limited either by the onset of arcing, by target melting, or by the limitations of the power supplies.

It is interesting to point out that the observed decrease in spoke mode number with increasing power is not always observed. The dynamic of HiPIMS patterns can differ from other self-organisation patterns from dielectric barrier discharge (DBD), gasdischarge semiconductor system (GDSS), cathode boundary layer discharge (CBLD). Benilov [40] postulated two competing mechanisms: electrostatic forces, favouring the appearance of modes with multiple spots, and charged particle diffusion favouring the appearance of modes with one spot. Due to their electrostatic nature, the multiple spots exhibit a repulsive interaction and the number of spots scales proportional to the voltage supplied.

The dynamic of the spoke mode transitions can be directly observed by monitoring the plasma using probe arrays in real time [41]. Figure 7 shows a measurement obtained by stacking the floating potential measurement of twelve azimuthally distributed flat probes on two of each other to show 7 revolutions of a spoke pattern on a 2" target. Two spokes are observed for discharge current from about $65 \mu$ s (discharge current $\mathrm{I}_{d}=$ $50 \mathrm{~A})$ to $105 \mu \mathrm{s}\left(\mathrm{I}_{d}=70 \mathrm{~A}\right)$. After $105 \mu \mathrm{s}$ the discharge current increases beyond $70 \mathrm{~A}$ and two spokes merge with the trailing spoke reducing in size and accelerating until it merges with the other leading spoke. After $120 \mu \mathrm{s}\left(\mathrm{I}_{d}=80 \mathrm{~A}\right)$ only one spoke remains, rotating along the racetrack. This merging of two spokes is explained by a competition between local Ar rarefaction at the location of a spoke and the replenishment of Ar behind the spoke along the racetrack: a spoke merger may occur with increasing target current when the larger local current through a leading spoke results in an enhanced local Ar rarefaction. In the wake of this larger spoke the Ar replenishment rate is reduced and the trailing spoke behind encounters a reduced neutral density. Consequently, the trailing spoke becomes smaller since fewer Ar neutrals are available to ionise and the $E \times B$ drift velocity becomes larger due to less friction with the neutral gas (in analogy to the spokes rotating faster at low pressures [3]). The trailing spoke, therefore, catches up to the leading spoke and both merge [41, 30].

As mentioned earlier, at peak power densities larger than $2 \mathrm{~kW} / \mathrm{cm}^{2}(\sim 70 \mathrm{~A}$ at 2 " planar target) the spokes disappear and the plasma becomes homogeneously distributed along the racetrack. Such transition can be clearly observed in figure 8 [17] since the 


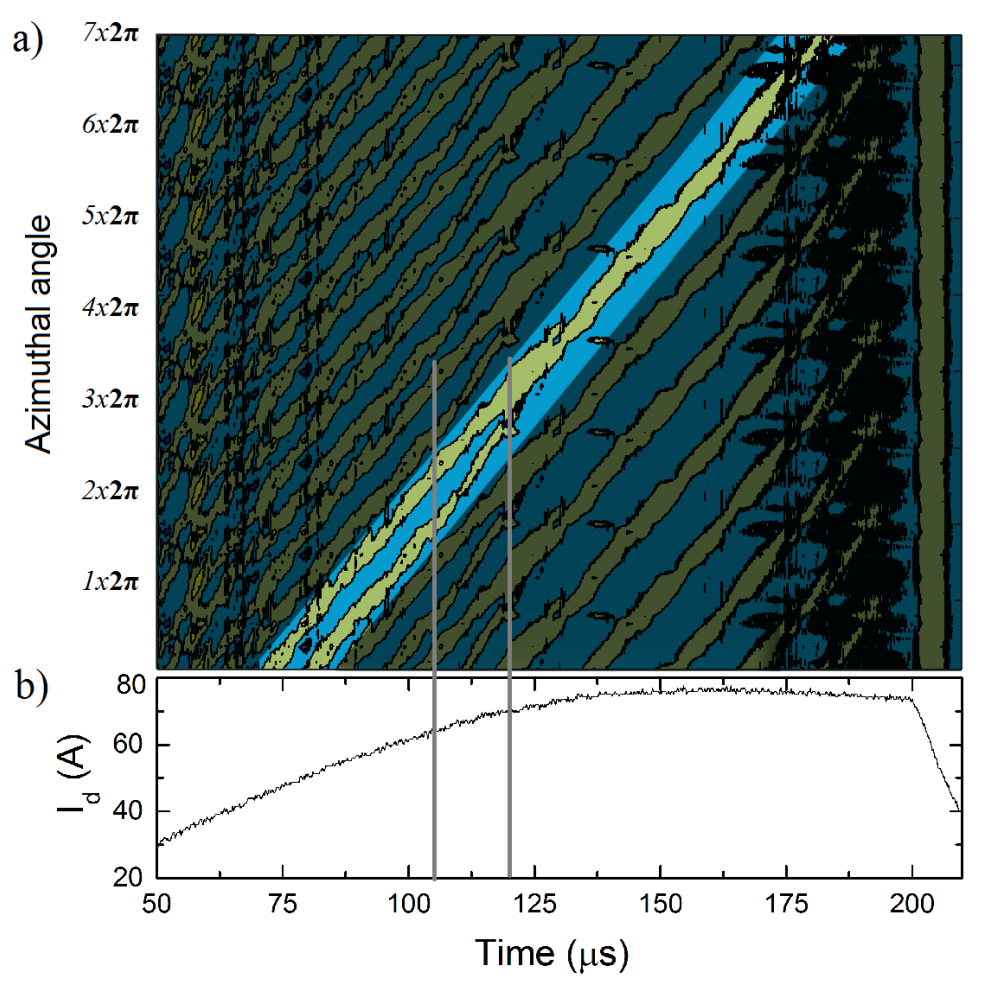

Figure 7. (a) Floating potential oscillations recorded using the 12-probe setup. HiPIMS discharge with $\mathrm{Cr}$ target at $0.5 \mathrm{~Pa}$. The light area emphasizes the transition between spoke mode 2 and spoke mode 1. (b) Discharge current waveform, adopted from [41].

floating potential oscillations due to the spokes passing by the probe disappear above a certain threshold. 
a)
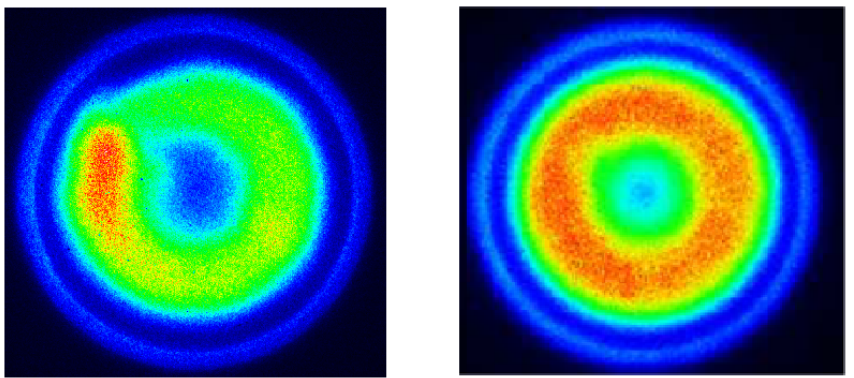

b)

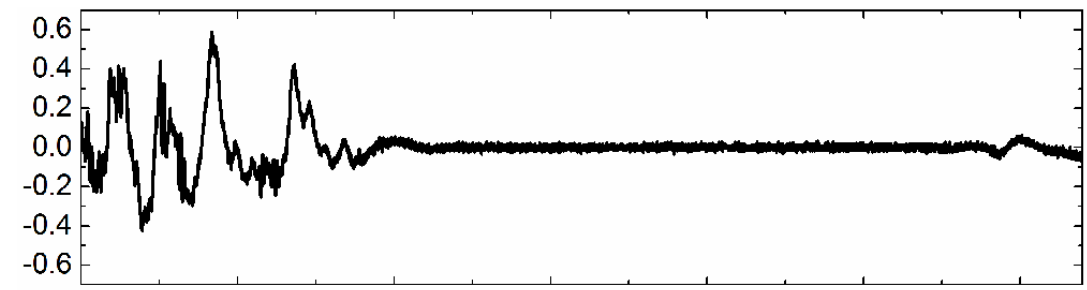

c)
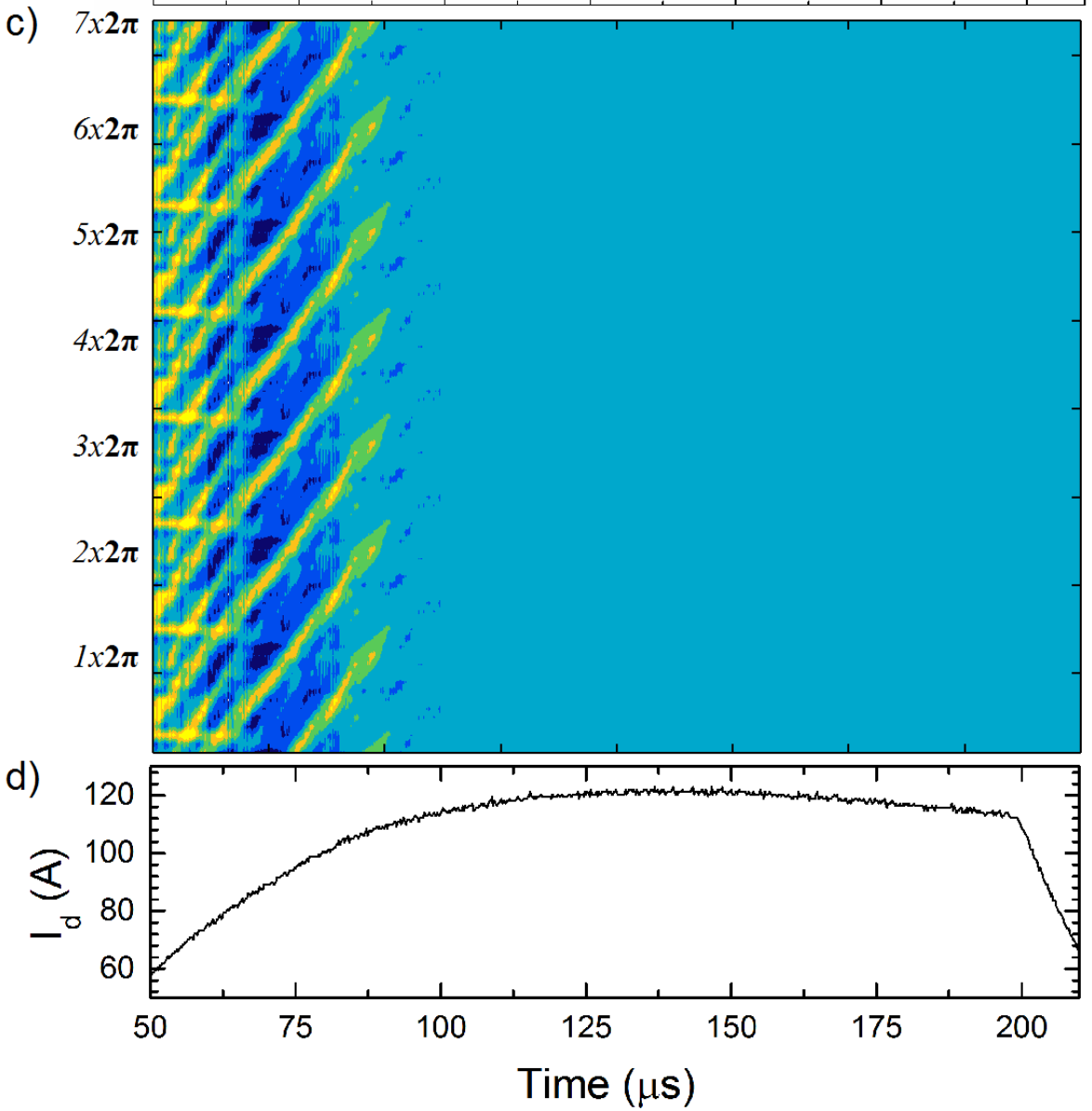

Figure 8. a) An ICCD image observed at comparable discharge conditions showing spoke mode 1 and homogeneous plasma. b) Probe 10 floating potential after EMD subtraction. c) Transition between the spoke modes and homogeneous plasma. The colour coding of the ICCD images and 2D floating potential map is different. d) Discharge current waveform. HiPIMS discharge, Cr target, adopted from [17]. 


\section{Spoke induced transport}

The spoke phenomenon and the dynamic of the HiPIMS plasmas are not only intriguing from a fundamental point of view, but are of paramount importance for the transport of the sputtered vapour from the target to the substrate as being at the core of many magnetron plasma applications. The transport of species is governed by the forces acting on them in three different regions: (i) atoms are sputtered at the target and enter the sheath and pre-sheath. Some of the sputtered atoms are ionised and may be attracted back to the target. This back-attraction may be enhanced by spokes due to associated azimuthal electric fields $E_{\phi}$ that trigger an $E_{\phi} \times B$ transport away from the target, but also towards the target leading to redeposition; (ii) atoms reach the the bulk plasma, where ionisation is dominant. The electrons are magnetically confined experiencing various drifts related to the $\mathbf{E} \times \mathbf{B}$ configuration. Due to quasi-neutrality, the electron and ion motion is bound by ambipolar fields. Again, spokes invoke internal electric fields $E_{\phi}$ that may lead to plasma flares and thus to anomalous electron transport; (iii) atoms and ions that escape the magnetic confinement region may arrive at the substrate. The energy and the flux of these species contributing to film growth depends directly on the plasma dynamic at the target and in the bulk plasma. All three regions are discussed in more detail in the following:

\subsection{Transport at the target}

The transport of plasma species close to the target is influenced by the electric fields surrounding a spoke and spoke rotation. The transport of plasma species along the racetrack is dominated by the $\mathbf{E} \times \mathbf{B}$ movement of the electrons. This transport, however, is modulated by the azimuthal electric fields $E_{\phi}$ surrounding each spoke. These azimuthal fields affect species transport threefold: (i) electrons that enter or leave a spoke along the racetrack become accelerated or decelerated; (ii) ions generated in a spoke are accelerated by these azimuthal fields $E_{\phi}$ to the perimeter of the plasma; (iii) an additional $E_{\phi} \times B$ movement of the electrons my lead to transport towards the target at the trailing edge and towards the substrate at the leading edge of a spoke. Ions, will follow this electron transport due to ambipolar electric fields. As a result, the ions may become re-deposited along the racetrack in the vicinity of a spoke.

The plasma density modulation associated with rotating spokes in HiPIMS has been investigated at the target surface using flush mounted inserts in the target at the racetrack position to monitor the local current density $[42,43,44]$. The travelling spokes that pass by such an insert cause regular perturbations in the local discharge current density of about $25 \%$ modulation are observed. It is interesting to note that the current at the target in between the spokes never reaches zero. Even though the emission patterns sometimes exhibit almost no emission in between the spokes (for example, figure 2d)) there are still charged particles present in between two spokes.

From the current density measurements, the plasma density at the sheath edge can

be estimated assuming a collisionless and non-magnetised sheath [44]. Both assumptions 
are valid for sheath thickness in the range of $100 \mu \mathrm{m}$, which is much smaller than the mean free path and the gyro-radius of electrons (and especially smaller than the gyroradius of the ions). The sheath thickness in HiPIMS discharge was also calculated using Child-Langmuir law, yielding values of about $100 \mu \mathrm{m}$ for target voltages corresponding to a power density above $250 \mathrm{~W} / \mathrm{cm}^{2}(\sim 10 \mathrm{~A}$ at 2 " planar target). This estimate is corroborated by a benchmark experiment using an optical inspection of the sheath thickness that yields values between $50 \mu \mathrm{m}$ and $100 \mu \mathrm{m}[42,44]$.

Assuming a rather constant electron temperature of $3 \mathrm{eV}$, it is possible to estimate the plasma density at the sheath edge from the Bohm velocity yielding values up to $9 \times 10^{19} \mathrm{~m}^{-3}$. This corresponds to a very high ionisation degree of $75 \%$, if one compares it to the cold background Ar density of $12 \times 10^{19} \mathrm{~m}^{-3}$. Such an estimate is only correct within $50 \%$, because Estrin et al. [43] showed that electron temperature may still vary between 2.1 and $3.9 \mathrm{eV}$.

The target atoms sputtered from the target are ionised in the cathode pre-sheath, and subsequently accelerated back to the target. Due to spoke rotation, some of the atoms or ions might be transported azimuthally by the spoke dynamic, and re-deposit at a distance with respect to the location where they were sputtered at the target surface. Layes et al. [45, 46] investigated this re-distribution of species using a marker technique: small inserts of $\mathrm{Cr}$ were embedded in an $\mathrm{Al}$ target at the racetrack position on a circular magnetron and exposed to a HiPIMS plasma. The HiPIMS discharge and the target surface composition were characterised in parallel for low, intermediate, and high power conditions, thus covering both the Ar-dominated and the metal-dominated HiPIMS regimes. The target surface composition was investigated intermittently by transferring the complete target in-vacuo to an adjacent photoelectron spectroscopy setup. The redeposition of sputtered species from the inserts was markedly more effective for Cr atoms than for $\mathrm{Al}$ atoms at all powers, with the target material predominantly transported in the $\mathbf{E} \times \mathbf{B}$ direction, irrespective of the presence of spokes. However, when spokes are present, an enhanced transport in the opposite $\mathbf{E} \times \mathbf{B}$ direction was observed. That was linked to the azimuthal electric fields $E_{\phi}$ at the sharp trailing edge of the spoke that may induce a stronger amiboplar $E_{\phi} \times B$ transport of plasma species back to the target.

\subsection{Transport in the bulk plasma}

The transport of species in the plasma bulk is governed by the magnetic confinement of the electrons. In order to conduct the current, the electrons have to travel across the magnetic field lines, which in classical representation is caused by electron-atom/ion collisions. Such cross-B diffusion is characterised by the so-called Hall $\omega_{c e} \tau$ parameter ( $\omega_{c e}$ - electron cyclotron frequency, $\tau$ - electron neutral collision time) yielding values around 16 for simple DC magnetron plasmas consistent with Bohm diffusion. However, in HiPIMS plasmas, it has been observed that the Hall parameter is in the range of only $2-5$, indicating a much faster diffusion of electrons across the magnetic field, referred to 
as anomalous diffusion.

The Hall parameter in HiPIMS was evaluated experimentally by comparing the Hall current and the discharge current [17]. In first approximation it is assumed that the ratio of these currents is comparable to the Hall parameter. Increasing the power from DC to HiPIMS plasma, the Hall parameter decreases to values of only 5 , when the power density is increased beyond $0.5 \mathrm{~kW} / \mathrm{cm}^{2}(\sim 20 \mathrm{~A}$ at 2 " planar target). The ICCD images showed stochastic plasma emission below $0.5 \mathrm{~kW} / \mathrm{cm}^{2}$, but self-organisation patterns above $0.5 \mathrm{~kW} / \mathrm{cm}^{2}$. If we assume that the charged particles diffuse within a spoke, being close to the target at the leading edge of the spoke, and being most distant to the target at the edge of the confinement zone at the trailing edge of the spoke, one can estimate the particle diffusion constants within a single spoke. Comparing the diffusion in the spoke and the average value of the Bohm diffusion coefficient one can estimate again a ratio of about 5 . This correlation implies that the dynamic of the plasma diffusion within a single spoke may in fact be correlated to the observed anomalous diffusion.

The plasma oscillations due to the presence of spokes exhibit typical frequencies in the range 100-400 kHz. In addition, oscillations in the $\mathrm{MHz}$ range have also been observed [1]. Lundin et al. [47] have associated these $\mathrm{MHz}$ oscillations with a modified two-stream instability (MTSI). The MHz oscillations observed by Tsikata et al. [48] using coherent Thomson scattering have been associated with the electron cyclotron drift instability (ECDI). They have associated the presence of a $\mathrm{MHz}, \mathrm{mm}$-scale instability, to a mm-scale electric field within the large-scale structures (spokes). The MHz oscillations have been correlated with the presence of collimated, axial electron density fluctuations at electron Larmor radius scales [48]. In addition to the observed large scale electric field structures, magnetic fluctuations have been found as well. The magnetic fluctuations are in the frequency range of $100 \mathrm{kHz}$, similar to the frequency of spoke rotation on 2" circular magnetrons. This relates the spoke phenomenon to also magnetically fluctuating structures that can grow with increasing $\beta$ parameter [49].

\subsection{Transport in the vicinity of the substrate}

Plasma species may escape the magnetic confinement region and travel towards the substrate. The electric and magnetic fields are very weak in this region and diffusion of the species is governed by collisions with the background gas and thermalised sputtered particles. The spoke dynamic may, nevertheless, influence the background plasma between the confinement region and the substrate, because, for example, the background plasma density may be enhanced by flares being ejected from the spokes.

At low powers typical for dcMS, spokes are Ar dominated and the plasma densities

are in the order of $10^{16} \mathrm{~m}^{-3}$, the ion energy distribution function (IEDF) of metal and Ar ions arriving at the substrate are similar, comprising low energy peak of thermalised species and a tail up to $10 \mathrm{eV}$ [50]. At high powers typical for HiPIMS, spokes are dominated by sputtered metal species, and the plasma densities in the spoke reach up 
to $8 \times 10^{19} \mathrm{~m}^{-3}$ [44]. The IEDF of metal ions consists of two main peaks at low energies (LE) as well as at high energies (HE) [51]: if the power density is increases to very high values so that the plasma becomes homogeneous again, the HE part of the metal ion IEDF does not change, but the LE peak is shifted by several $\mathrm{eV}$ to higher energies. This has been attributed to a reduction of low energy Ar particles in the target vicinity as the homogeneous plasma is considered to be a sign of full self-sputtering regime.

The IEDFs are also measured for ions ejected in radial direction to the HiPIMS plasma showing a difference in $\mathbf{E} \times \mathbf{B}$ compared to the retrograde $\mathbf{E} \times \mathbf{B}$ direction $[52,53]$. The low energy peak stays constant but the number of ions within high energy tail of IEDF measured in $\mathbf{E} \times \mathbf{B}$ direction is always higher compared to IEDF measured in retrograde $\mathbf{E} \times \mathbf{B}$ direction. Measurements performed at dcMS conditions also show a pronounced asymmetry in the target plane, suggesting that similar mechanisms may be applicable when the discharge is continuously operated, even though the rotation of the spokes is in retrograde $\mathbf{E} \times \mathbf{B}$ direction.

The IEDF measurements for conditions with and without ionisation zones, measured for ions ejected radially to the magnetron [54], revealed that ion energies are generally lower and the pronounced azimuthal asymmetry disappears when ionisation zones are absent.

Finally, the ion-to-metal-flux-fraction (IMFF) in the growth flux arriving at the substrate has been measured by Biskup et al. [55] using a combination between a retarding field analyser covering a quartz microbalance. It was shown that the growth rate per invested power significantly decreases with increasing HiPIMS pulse power due to the return effect close to the target. However, this decrease in growth rate was mitigated, when spokes form in the HiPIMS plasmas. This can be explained by the additional electric fields surrounding the spokes that support the transport of ions towards the substrate. The IMFF values continuously increased with increasing peak power density up to values close to $80 \%$, indicating film growth by incident ions only at very higher powers. 


\section{Spokes as regions of high electrical potential}

The theoretical description of the origin of spokes is very challenging due to the complexity of the phenomenon involving gas rarefaction, secondary electron generation and instabilities in the strong gradients of magnetic field and plasma density. Especially the observation of very specific energies of ions arriving at the substrate led to the hypothesis of the spokes in high power impulse magnetron sputtering being regions of a locally enhanced electrical potential. This has been postulated by Maszl at al. [56] in relation to the potential hump hypothesis by Anders et al. [57]. Such potential structures can explain the disruption of the confined electron trajectories, the formation of plasma flares, the relatively high energy of ions at the substrate, and the difference of ion energy distribution functions for radially ejected ions when the $\mathbf{E} \times \mathbf{B}$ is compared to the $-\mathbf{E}$ $\times \mathbf{B}$ direction. The existence of an enhanced electrical potential at the location of the spoke is corroborated by several observations:

- (i) Measurement of the plasma potential distribution above the target in dcMS plasmas: spokes are also observed in low power dcMS magnetrons where the plasmas are accessible by Langmuir probes. Cold and hot emissive probes have been used to measure the electric fields and net space charge distributions travelling with the spoke along the target racetrack by Panjan et al. [58], as illustrated in Fig. 9. The leading edge of the spoke is characterised by an electric double layer, energising drifting electrons, which can further cause ionisation and excitation. Before crossing the double layer, electrons are at a low electrical potential, while within the spoke beyond the double layer, they are at a high electrical potential. Consequently, electrons created in the plasma are capable of gas ionisation once they cross and obtain energy from the azimuthal electric fields $E_{\phi}$ surrounding a spoke. The electrical potential is very asymmetric, showing a steep rise at the front edge and then a gradual slope towards the trailing edge. Electrons drifting in the $\mathbf{E}$ $\times \mathbf{B}$ direction from the denser plasma of the ionisation zone into a region of lower plasma density cause a slight imbalance of charge, with an increasingly negative net charge forming. A large charge imbalance around the leading edge of the ionisation zone is sustained by a strong azimuthal electric field $E_{\phi}$ that accelerates the ions in lateral direction, but also towards the target or the substrate when the follow by ambipolar fields the additional $E_{\phi} \times B$ movement of the electrons. The space charge and related field structure can explain many observations, including the ionisation zone motion and the asymmetry in the IEDFs of the radially ejected ions. The authors claim that although these measurements and their interpretation are related to dcMS, many of the features may also be associated with spokes in HiPIMS.

- (ii) Measurement of the plasma density at the target sheath, and at $15 \mathrm{~mm}$ above the target surface: Measurements using circular inserts in the target showed that the plasma density increases linearly with power density, exhibiting a $25 \%$ modulation induced by the travelling spokes [44]. The average plasma density was in the range 


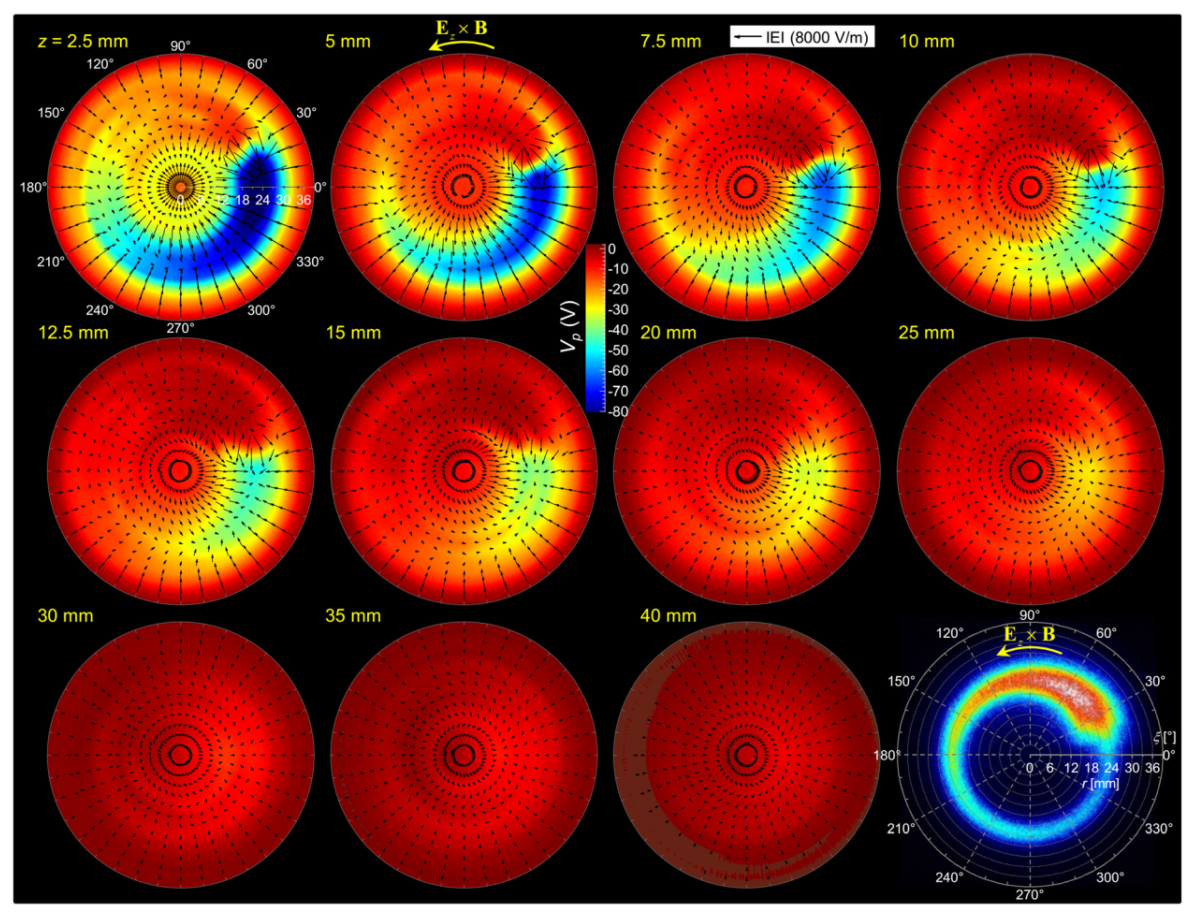

Figure 9. Plasma potential (color) and electric field (vector) distributions in the n-r plane for different axial distances from the target surface $(\mathrm{z}=2.540 \mathrm{~mm})$, adopted from [58]. Magnetron is 3" round planar magnetron.

of $10^{19} \mathrm{~m}^{-3}$. At $15 \mathrm{~mm}$ above the target, spokes have also been monitored by Estrin et al. [43] using probes. They observed that the spokes have a higher electron densities and lower electron temperatures at their leading edge $\left(n_{e} \simeq 2.0 \times 10^{19}\right.$ $\mathrm{m}^{-3}, T_{e} \simeq 2.1 \mathrm{eV}$ ), but lower electron densities and higher electron temperatures at their trailing edge $\left(n_{e} \simeq 10^{19} \mathrm{~m}^{-3}, T_{e} \simeq 3.9 \mathrm{eV}\right)$. A calculation of the plasma potential $V_{p}$ based on the floating potential $V_{f}$ and $T_{e}$ measurements showed that the spokes correspond to a positive potential of up to $+9 \mathrm{~V}$ relative to the electrical potential in between the spokes. However, the regions of high electrical potential are slightly shifted with respect to the region of maximum electron density $n_{e}$. Azimuthal electric fields $E_{\phi}$ of the order of $1 \mathrm{kV} / \mathrm{m}$ are deduced from the electrical potential gradients along the ractrack.

- (iii) Ion energy distribution function (IEDF) of metal ions with an peak at energies in the range 15-25 eV: As an example, the ion energy distributions in HiPIMS using a Ti target have been measured by Maszl et al.[56]. The IEDF of Ar ions consists of a low energy (LE) peak and a tail stretching up to $10 \mathrm{eV}$. The IEDF of $\mathrm{Ti}^{+}$ions consists of a low energy (LE) peak and, at high power densities where the metal dominated spokes are present, an additional peak at high energies (HE) in the range $15-25 \mathrm{eV}$ is observed. The position of the HE peak increases with target power density. This observation can easily be explained by assuming that the HE group of ions originate from the high electrical potential in the spoke. These ions are accelerated in the electric fields surrounding a spoke and travel 
towards the substrate. Additionally, it was found that doubly charged ions exhibit approximately twice the energy of singly charged ions, indicating the validity of an electric acceleration mechanisms.

- (iv) High diffusion coefficients: The observation of cross-field electron diffusion a few times higher than the Bohm diffusion indicates that ions from the spoke are accelerated by the potential structure away from the target. Therefore, in the rotating spoke, the electric field of surrounding a spoke acts as a plough displacing ions in all directions [17]. 


\section{Explaining the spoke phenomenon}

The theoretical description of spokes is still a matter of debate. One may distinguish either phenomenological single particle descriptions, fluid descriptions based on plasma equilibria, kinetic simulations, or equivalent simulations for Hall thrusters:

- (i) phenomenological single particle descriptions: a phenomenological explanation of the spoke phenomenon corresponding to a hump in the electrical potential surrounded by double layers has been postulated by Anders et al. [57]. According to this description, the electrical fields surrounding a spoke cause an acceleration and deceleration of electrons that enter or leave this region. Depending on the ionisation rates, a local variation in electron density occurs. Since the $\mathbf{E} \times \mathbf{B}$ drift of the electrons is 10 times faster than the actual spoke movement, the drifting electrons that enter a spoke are deflected towards the target and become reflected at the sheath potential. These reflected electrons are energised as they enter a region of higher electrical potential. This enables a feedback mechanism for maintaining and amplifying the ionisation in the spoke. An enhanced electrical potential results from the inertia of ions that are left behind as a fraction of electrons escapes from the ionisation zone. Since inelastic collisions of energetic electrons are the origin for light emission, the observation of bright spokes is an indication for high electron energies locally.

- (ii) fluid descriptions: plasma instabilities fall into to two classes of fluid and kinetic instabilities. The spoke phenomenon belongs to a low frequency instability where the fluid description is still appropriate. Based on the topology of a magnetron plasma and the gradients in electron density and magnetic field, the spoke phenomenon can be related to the Simon-Hoh instability $[9,8]$, that occurs above a specific density gradient for a given magnetic and electric field. Since the spoke phenomenon is associated with strong plasma density gradients locally, the triggering of these instabilities is very likely. The propagation of the corresponding drift waves depends then on the direction of the density gradients and the directions of the magnetic and electric fields. Since the electric field surrounding a spoke may point towards the target in the magnetic pre-sheath, but also towards the substrate in the region between spoke and substrate, the group velocity of the spokes phenomenon may point either clockwise or counter-clockwise on a circular target, depending whether the $E$-field towards the target or the $E$-field towards the substrate dominates the driving $\mathbf{E} \times \mathbf{B}$ motion [59]. This may resolve also the mystery regarding the $\mathbf{-} \mathbf{E} \times \mathbf{B}$ rotation at low plasma powers: the retrograde motion is defined with respect to the $E_{\text {pre-sheath }}$-field in the pre-sheath pointing towards the target. If, however, $E_{\text {pre-sehath }}$ is not dominating the transport, but rather the field $E_{\text {spoke }}$ pointing in the opposite direction from the spoke to the substrate, a reverse of the rotation direction results. Such a motion is an ordinary $\mathbf{E} \times \mathbf{B}$ motion, only dominated by $E_{\text {spoke }}$ rather than by $E_{\text {pre-sheath. }}$ A conclusive description is, however, not yet possible, because the linear instability analysis following the 
Simon-Hoh scheme is based on small perturbations, whereas the experiment show density modulations of at least $25 \%$. In addition, fluid description do not capture secondary electron effects or gas rarefaction which may have also an influence on the spoke phenomenon.

- (iii) kinetic descriptions: A self-consistent kinetic simulation has been presented by Boeuf et al. [60] showing the evidence of the formation of a rotating ionisation front (spoke) that describe experiments related to the $v_{C I V}$ very well. The rotating structures in the simulations originate from a current that is driven across a uniform magnetic field by an applied voltage. The plasma is azimuthally separated into two regions at different electric potentials, with a sharp potential drop in between that defines an azimuthal ionisation front (spoke) that propagate in the direction of the higher electrical potential. The $\mathbf{E} \times \mathbf{B}$ electron current is subject to a twostream instability. The net transport of the electrons is assured in a region of lower electrical potential where the azimuthal electric field $E_{\phi}$ induces a $E_{\phi} \times B$ drift towards the substrate. These results provide a new insight in the physics of plasma transport in cross-field devices and suggest that the rotating instabilities observed in the relatively complex and high-current plasma experiments designed in the context of CIV studies, should also be observed in much simpler conditions such as those of low current cylindrical magnetron discharges.

In a follow up paper, Boeuf et al. [61] studied the electron transport in three typical but simplified $\mathbf{E} \times \mathbf{B}$ configurations of low temperature plasma sources on the basis of results from PIC-MCC simulations. Electron vortices are observed in the simulations for low pressure magnetron discharges (typical of Penning or magnetron gauges), as well as rotating spokes and azimuthal electron-cyclotron drift instability (ECDI). Especially the ECDI can only be capture in kinetic simulations and constitute a high frequency phenomenon, which contribute significantly to the anomalous transport of electrons through a magnetic barrier. Fully 3D kinetic PIC simulations, however, are still difficult to realise, because the simulation of very large plasma densities as being typical for HiPIMS is still beyond the capabilities of the computer hardware.

Finally, Revel et al. used Pseudo3D modeling [62] to analyse the nature of spokes by invoking a fixed spoke region on a linear section of a rectangular magnetron. the simulation of the dynamic of this given spoke region reveals electron excursions near the racetrack, spoke self-polarisation, and a non-uniform ionisation within spokes. Such pseudo3D approaches may be the basis for more advanced phenomenological models.

- (iv) kinetic and fluid descriptions of Hall thrusters: many of simulations are performed to model Hall thrusters which exhibit a similar magnetic field topology compared to HiPIMS magnetrons. These simulations simplify the geometry and focus on the instabilities in the plasma that develop due to plasma motion in crossed electric and magnetic fields. Janes et al. [5] investigated correlations between density and electric fields in connection to the observed anomalous cross-B 
diffusion. They concluded that the effective electron diffusion can be attributed to the lowest frequency component such as spoke rotation.

Similar conclusions were reached by Janhunen et al. [63] who used particlein-cell simulation in 1D3V to study the electron-cyclotron instability driven by the electron $\mathbf{E} \times \mathbf{B}$ current. The electron-cyclotron instability (ECDI) is based on the interaction of the electron cyclotron mode with ion plasma oscillations. The simulations have shown that the ion-sound like regime of the ECDI (with fully demagnetised electrons) does not occur for plasma parameters relevant for magnetron and Hall thruster operation. Their simulation showed further that electron dynamics and the anomalous current is dominated by long wavelength and low frequency modes (the lower hybrid range and below). This is consistent with the experimental observations of spoke induced transport in Hall thrusters [64].

A similar conclusion was presented by Lakhin et al [65] who used a two-fluid model with finite electron temperature in an inhomogeneous magnetic field. They showed that the electron inertia and finite Lamor radius effects act as a stabilising factor of the short-wavelength fluctuations. In some cases they have found that such effects completely suppress the high-frequency short-wavelength modes leaving only the long-wavelength low-frequency modes unstable.

Koshkarov et al. [66] investigated current flow instabilities and nonlinear structures in dissipative two-fluid plasmas. The current flow instability occurs due to a positive feedback between the electron and ion current coupled by quasi-neutrality. This type of instabilities occurs for modes propagating along the direction of the current flow, without requiring a density gradient that is needed for Simon-Hoh instabilities $[8,9]$. They concluded that the current flow instabilities play a dominant role in breathing oscillations.

Numerical analysis of azimuthally rotating spokes in a crossed-field discharge plasma and comparison with other simulations have been performed by Kawashima et al. [67]. The comparison of their modelled phase velocities with predictions of Esipchuck [68] and Frias [69] showed comparable results within $10 \%$. The corresponding dispersion relations are both based on the drift instability resulting from gradients in plasma density and magnetic field. Thus, it has been concluded that the drift instability is the driving mechanism of rotating spokes. Kawashima et al. separated the development of the instability in three stages: (i) at the beginning, the plasma is homogeneously distributed in azimuthal direction, with a small plasma wave propagating in $\mathbf{E} \times \mathbf{B}$ direction. The amplitude of the plasma wave grows linearly due to the gradient drift instability. The linear growth results in fluctuations of plasma density and potential; (ii) the azimuthal fluctuations in the plasma density causes non-uniformities in the ionisation rate and the neutral atom density, which in turn enhances the non-uniformity of plasma density. The non-uniform ionisation profile propagates also in axial direction; (iii) after a certain time, non-uniformities in the azimuthal electrical field $E_{\phi}$ and neutral atom density 
no longer grow, due to the boundary conditions of a fixed electrical potential at the cathode and anode as well as a limited neutral atom flow rate. Finally, the instability saturates and the non-uniformity of the plasma constituents becomes fully developed. A coherent structure of an azimuthal wave pattern is formed and propagates in the azimuthal direction with a constant phase velocity. After the gradient drift instability saturates, the propagation velocity of the coherent structure is close to the phase velocity of the gradient drift instability. This indicates that the azimuthal transport of the coherent wave is driven by the gradient drift wave, whereas the non-uniform ionisation plays an important role in the wave amplication and the transport of plasma in the axial direction towards the substrate.

Summarizing, one may state that the theoretical explanation of the spoke phenomenon is still challenging because all approaches so far have their limitations: the phenomenological models depend on assumptions regarding the topology of the spoke structure and the surrounding fields as well as regarding the magnitudes of ionisation and drifts, the fluid models do not cover gas depletion and secondary electron generation, and the kinetic simulations can only model rather low density plasmas. Nevertheless, all these descriptions already provide very valuable insight into the spoke phenomenon and the quantitative description and prediction of the spoke phenomenon seems to be in reach within the next years. 


\section{Conclusion}

Over the recent years, great progress in the understanding of the spoke phenomenon in HiPIMS plasmas has been achieved in the scientific community. Based on experimental data using Langmuir, emissive and floating probes at the perimeter of the rotating spoke, oscillations in the plasma potential by $10 \mathrm{~V}$ have been monitored. By using inserts in the target of a magnetron plasma, the electron density at the sheath edge had been determined in the range of $10^{20} \mathrm{~m}^{-3}$ with fluctuations in the range of $30 \%$. By using optical emission spectroscopy, the emission patterns of the spoke phenomenon was used to characterise the main parameters such as spoke movement with velocities in the range of $\mathrm{km} / \mathrm{s}$ and a direction which reverses with increasing plasma power. The shape of the spoke depends on the target material and on the state of target poisoning. From ion energy and mass spectrometry measurement the occurrence of an extra group of energetic ions, at energies around $20 \mathrm{eV}$ at the substrate position has been observed. Such energetic ions are also observed in radial direction to the magnetron target.

On the basis of all this experimental evidence, a robust and at least phenomenological model of an electrical potential maximum in the region of the spoke has been identified. The local maximum in the electrical potential implies electric fields surrounding this spoke, which accelerate the ions and which may dominate the direction of the drift waves and thus the movement of the spokes. Any quantitative and more direct information about the inside of a spoke is still difficult to achieve due to the incompatibility of probe diagnostics with the high thermal load inside a spoke. In addition, non-invasive emission spectroscopy, as an alternative, is also difficult to interpret due to the complex plasma topology, which would require extensive collisional radiative modelling. The theoretical modelling and simulation is also hampered by the limited capability of 3D PIC codes, which, at the movement, can only model rather low density plasmas. However, all these challenges will be mastered in the coming years so that, based on a better and quantitative understanding, the design and thus the performance of HiPIMS plasmas can be significantly improved in the future. 


\section{Acknowledgements}

This work has been supported by the German Science Foundation (DFG) within the frame of the collaborative research centre SFB-TR 87.

\section{References}

[1] Winter J, Hecimovic A, de los Arcos T, Böke M and Schulz-von der Gathen V 2013 Journal of Physics D: Applied Physics 46084007 ISSN 0022-3727, 1361-6463

[2] Kozyrev A V, Sochugov N S, Oskomov K V, Zakharov A N and Odivanova A N 2011 Plasma Physics Reports 37 621-627 ISSN 1063-780X, 1562-6938

[3] Ehiasarian A P, Hecimovic A, de los Arcos T, New R, Schulz-von der Gathen V, Böke M and Winter J 2012 Applied Physics Letters 100114101 ISSN 0003-6951

[4] Anders A 2012 Applied Physics Letters 100224104 ISSN 0003-6951, 1077-3118

[5] Janes G S 1966 Physics of Fluids 91115 ISSN 00319171

[6] De la Rue W and Muller H W 1878 Philosophical transactions of the Royal Society of London $\mathbf{1 6 9}$ $155-241$

[7] Hayashi T, Sato T and Complexity Simulation Group 1999 Plasma Physics and Controlled Fusion 41 A229-A238 ISSN 0741-3335

[8] Simon A 1963 Physics of Fluids 6382 ISSN 00319171

[9] Hoh F C 1963 Physics of Fluids 61184 ISSN 00319171

[10] Brenning N, Merlino R L, Lundin D, Raadu M A and Helmersson U 2009 Physical Review Letters 103 ISSN 0031-9007, 1079-7114

[11] Thornton J 1978 J. Vac. Sci. Technol. A 15171

[12] Sheridan T E and Goree J 1989 Journal of Vacuum Science E Technology A: Vacuum, Surfaces, and Films 7 1014-1018 ISSN 0734-2101, 1520-8559

[13] Martines E, Cavazzana R, Serianni G, Spolaore M, Tramontin L, Zuin M and Antoni V 2001 Physics of Plasmas 8 3042-3050 ISSN 1070-664X

[14] Martines E, Zuin M, Antoni V, Cavazzana R, Serianni G, Spolaore M and Nakashima C 2004 Physics of Plasmas 11 1938-1946

[15] Hecimovic A, Böke M and Winter J 2014 Journal of Physics D: Applied Physics 47102003 ISSN $0022-3727$

[16] Andersson J, Ni P and Anders A 2013 Applied Physics Letters 103054104 ISSN 0003-6951, 10773118

[17] Hecimovic A 2016 Journal of Physics D: Applied Physics 49 18LT01 ISSN 0022-3727

[18] Hecimovic A, Britun N, Konstantinidis S and Snyders R 2017 Applied Physics Letters 110014103

[19] Ni P A, Hornschuch C, Panjan M and Anders A 2012 Applied Physics Letters 101 224102-224102-5 ISSN 00036951

[20] Anders A 2017 Journal of Applied Physics 121171101 ISSN 0021-8979, 1089-7550

[21] Hecimovic A, Corbella C, Maszl C, Breilmann W and von Keudell A 2017 Journal of Applied Physics 121171915

[22] Marcak A, Corbella C, de los Arcos T and von Keudell A 2015 Review of Scientific Instruments 86106102 ISSN 0034-6748, 1089-7623

[23] Shayestehaminzadeh S, Arnalds U B, Magnusson R L and Olafsson S 2015 AIP Advances 5117240

[24] Hnilica J, Klein P, Šlapanská M, Fekete M and Vašina P 2018 Journal of Physics D: Applied Physics 51095204 ISSN 0022-3727

[25] Preissing S 2016 Spectroscopic Investigation of an Extensive Magnetron Plasma Master thesis Ruhr University Bochum Bochum

[26] Anders A and Yang Y 2017 Applied Physics Letters 111064103 
[27] Bobzin K, Brögelmann T, Kruppe N C, Engels M, von Keudell A, Hecimovic A, Ludwig A, Grochla D and Banko L 2017 Journal of Applied Physics 122015302 ISSN 0021-8979

[28] Anders A and Yang Y 2018 Journal of Applied Physics 123043302 ISSN 0021-8979

[29] Yang Y, Zhou X, Liu J X and Anders A 2016 Applied Physics Letters 108034101 ISSN 00036951

[30] Klein P, Estrin F L, Hnilica J, Vašina P and Bradley J W 2017 Journal of Physics D: Applied Physics 50015209 ISSN 0022-3727

[31] de los Arcos T, Schröder R, Gonzalvo Y A, der Gathen V S v and Winter J 2014 Plasma Sources Science and Technology 23054008 ISSN 0963-0252, 1361-6595

[32] de los Arcos T, Layes V, Gonzalvo Y A, der Gathen V S v, Hecimovic A and Winter J 2013 Journal of Physics D: Applied Physics 46335201 ISSN 0022-3727

[33] Anders A, Ni P and Rauch A 2012 Journal of Applied Physics 111053304 ISSN 0021-8979, 10897550

[34] Panjan M, Loquai S, Klemberg-Sapieha J E and Martinu L 2015 Plasma Sources Science and Technology 24065010 ISSN 0963-0252

[35] Gallian S, Trieschmann J, Mussenbrock T, Brinkmann R P and Hitchon W N G 2015 Journal of Applied Physics 117023305 ISSN 00218979

[36] Ziegler J F, Ziegler M D and Biersack J P 2010 Nuclear Instruments and Methods in Physics Research Section B: Beam Interactions with Materials and Atoms 268 1818-1823 ISSN 0168$583 \mathrm{X}$

[37] Depla D, Buyle G, Haemers J and De Gryse R 2006 Surface and Coatings Technology 2004329 4338 ISSN 0257-8972

[38] Brenning N, Lundin D, Minea T, Costin C and Vitelaru C 2013 Journal of Physics D: Applied Physics 46084005 ISSN 0022-3727, 1361-6463

[39] Martines E, Zuin M, Cavazzana R, Adámek J, Antoni V, Serianni G, Spolaore M and Vianello N 2014 Physics of Plasmas 21102309 ISSN 1070664X

[40] Benilov M S 2008 Physical Review E 77 ISSN 1539-3755, 1550-2376

[41] Hecimovic A, Schulz-von der Gathen, V, Böke M, von Keudell A and Winter J 2015 Plasma Sources Science and Technology 24045005 ISSN 0963-0252

[42] Poolcharuansin P, Estrin F L and Bradley J W 2015 Journal of Applied Physics 117163304 ISSN 0021-8979

[43] Estrin F L, Karkari S K and Bradley J W 2017 Journal of Physics D: Applied Physics 50295201 ISSN 0022-3727

[44] Hecimovic A, Held J, der Gathen V S v, Breilmann W, Maszl C and von Keudell A 2017 Journal of Physics D: Applied Physics 50505204 ISSN 0022-3727

[45] Layes V, Monje S, Corbella C, Trieschmann J, de los Arcos T and von Keudell A 2017 Applied Physics Letters 110081603 ISSN 0003-6951

[46] Layes V, Monje S, Corbella C, Schulz-von der Gathen V, von Keudell A and de los Arcos T 2017 Journal of Applied Physics 121171912 ISSN 0021-8979

[47] Lundin D, Helmersson U, Kirkpatrick S, Rohde S and Brenning N 2008 Plasma Sources Science and Technology 17025007 ISSN 0963-0252

[48] Tsikata S and Minea T 2015 Physical Review Letters 114185001

[49] Spagnolo S, Zuin M, Cavazzana R, Martines E, Patelli A, Spolaore M and Colasuonno M 2016 Plasma Sources Science and Technology 25065016 ISSN 0963-0252

[50] Bohlmark J, Lattemann M, Gudmundsson J, Ehiasarian A, Aranda Gonzalvo Y, Brenning N and Helmersson U 2006 Thin Solid Films 515 1522-1526 ISSN 00406090

[51] Breilmann W, Eitrich A, Maszl C, Hecimovic A, Layes V, Benedikt J and von Keudell A 2015 Journal of Physics D: Applied Physics 48295202 ISSN 0022-3727

[52] Panjan M, Franz R and Anders A 2014 Plasma Sources Science and Technology 23025007 ISSN 0963-0252

[53] Franz R, Clavero C, Kolbeck J and Anders A 2016 Plasma Sources Science and Technology 25 015022 ISSN 0963-0252 
[54] Yang Y, Tanaka K, Liu J and Anders A 2015 Applied Physics Letters 106124102 ISSN 00036951

[55] Biskup B, Maszl C, Breilmann W, Held J, Böke M, Benedikt J and von Keudell A 2018 Journal of Physics D: Applied Physics 51115201 ISSN 0022-3727, 1361-6463

[56] Maszl C, Breilmann W, Benedikt J and von Keudell A 2014 Journal of Physics D: Applied Physics 47224002 ISSN 0022-3727

[57] Anders A, Panjan M, Franz R, Andersson J and Ni P 2013 Applied Physics Letters 103144103 ISSN 0003-6951, 1077-3118

[58] Panjan M and Anders A 2017 Journal of Applied Physics 121063302

[59] Hecimovic A, Maszl C, Schulz-von der Gathen, V, Böke M and von Keudell A 2016 Plasma Sources Science and Technology 25035001 ISSN 0963-0252

[60] Boeuf J P and Chaudhury B 2013 Physical Review Letters 111155005

[61] Boeuf J P 2014 Frontiers in Physics 2 ISSN 2296-424X

[62] Revel A, Minea T and Tsikata S 2016 Physics of Plasmas 23100701 ISSN 1070-664X

[63] Janhunen S, Smolyakov A, Chapurin O, Sydorenko D, Kaganovich I and Raitses Y 2017 Physics of Plasmas 25011608 ISSN 1070-664X

[64] Parker J B, Raitses Y and Fisch N J 2010 Applied Physics Letters 97091501 ISSN 0003-6951

[65] Lakhin V P, Ilgisonis V I, Smolyakov A I, Sorokina E A and Marusov N A 2018 Physics of Plasmas 25012106 ISSN 1070-664X

[66] Koshkarov O, Smolyakov A I, Romadanov I V, Chapurin O, Umansky M V, Raitses Y and Kaganovich I D 2017 Physics of Plasmas 25011604 ISSN 1070-664X

[67] Kawashima R, Hara K and Komurasaki K 2018 Plasma Sources Science and Technology 27035010 ISSN 0963-0252

[68] Esipchuck Y B and Tilinin G 1976 Sov. Phys. Tech. Phys. 21417

[69] Frias W, Smolyakov A I, Kaganovich I D and Raitses Y 2012 Physics of Plasmas 19072112 ISSN 1070-664X, 1089-7674 